\title{
Three-Territory DWI Acute Infarcts: Diagnostic Value in Cancer-Associated Hypercoagulation Stroke (Trousseau Syndrome)
}

\author{
(D)P.F. Finelli and (D) A. Nouh
}

\begin{abstract}
BACKGROUND AND PURPOSE: DWI infarcts involving the bilateral anterior and posterior circulation suggest an embolic etiology. In the absence of an identifiable embolic source, we analyzed DWI lesions involving these 3 cerebral territories to determine the diagnostic value for ischemic infarction caused by cancer-associated hypercoagulation.
\end{abstract}

MATERIALS AND METHODS: A retrospective analysis of all brain MR imaging studies at our institution from July 2014 to June 2015 was conducted, yielding 4075 studies. Of those, 17\% $(n=709)$ contained the terms "restricted-diffusion" plus either "numerous," "innumerable," "multiple," or "bilateral." Of these 709 reports, $6 \%(n=41)$ of DWI lesions involving 3 or more vascular territories of the bilateral anterior and posterior circulation were analyzed.

RESULTS: Of the 41 patients, 19 separate etiologies were identified, the most frequent being malignancy-related infarctions (22\% [ $n=9]$ ) and hypoxic-ischemic injury (12\% $[n=5])$. Only 2 patients had an indeterminate etiology. The most frequent etiology of infarctions not suspected clinically or radiographically was malignancy $(P<.001)$. Infarctions of malignancy had a characteristic appearance, being nonenhancing, nonring-appearing clusters or single areas of restricted diffusion of $0.5-2 \mathrm{~cm}$ with a peripheral location or larger vascular territories, uncommonly in a watershed distribution, and with absence of diffuse cortical ribbon or deep gray nuclei involvement.

CONCLUSIONS: Approximately 1 in 5 ischemic infarcts in patients with DWI lesions involving 3 vessel territories are malignancy related. In the absence of an identifiable embolic source, ischemic infarction with cancer-associated hypercoagulation accounts for $75 \%$ of cases. Cancer-associated hypercoagulation infarction should be considered, particularly when no other cause is apparent.

ABBREVIATION: TS $=$ Trousseau syndrome

$\mathbf{U}^{\mathrm{n}}$ to $15 \%$ of patients with malignancy may experience a thromboembolic cerebrovascular event during their clinical course. ${ }^{1}$ In addition, malignancy is frequently overlooked as a cause of stroke and is commonly undiagnosed until a second event occurs. ${ }^{2}$ Though the paraneoplastic hypercoagulable state is complex and not fully understood, it is an established mechanism of thrombosis in malignancy. The importance of diagnosing cancer-associated hypercoagulation is appreciated because it may be the heralding manifestation of occult malignancy. Treatment with heparin has been demonstrated effective in preventing thrombotic events, including stroke. ${ }^{3,4}$

Trousseau syndrome (TS) is a hypercoagulable state, associ-

Received March 23, 2016; accepted after revision April 28.

From the Department of Neurology, Hartford Hospital and University of Connecticut School of Medicine, Hartford, Connecticut.

Please address correspondence to Pasquale F. Finelli, MD, Department of Neurology, Hartford Hospital and University of Connecticut School of Medicine, 80 Seymour St, Hartford, CT 06102-5037; e-mail: pasquale.finelli@hhchealth.org

http://dx.doi.org/10.3174/ajnr.A4846 ated with cancer, that includes various disorders probably involving multiple overlapping mechanisms. It has been suggested the term "Trousseau syndrome" be restricted to unexplained thrombotic events that either precede the diagnosis of an occult visceral malignancy or appear concomitantly with the tumor. ${ }^{5}$ Cerebral infarction, mostly caused by in situ thrombosis in medium and small vessels, is thought to be related to the prothrombic state of TS. Verrucous endocarditises associated with cerebral emboli, infection, or therapy-related strokes are alternative causes of ischemic infarction. ${ }^{2,6}$ Given the familiar usage of TS by some authors to refer to cancer-associated hypercoagulation, ${ }^{5}$ we use TS in that context in this discussion.

DWI primarily defines ischemic infarcts in malignancy as small and involving multiple vessel territories, ${ }^{6-9}$ with the number of territories involved correlating with the likelihood of this syndrome. ${ }^{4,10-12}$ However, studies specifically evaluating MR imaging in cerebral infarction with TS and its diagnostic value in establishing causality are lacking. Distinct from prior reports where patient selection was based on the presence of stroke with malig- 
Etiologies of 3 territory diffusion-weighted lesions

\begin{tabular}{|c|c|c|}
\hline & No. & Clinical/MR Imaging, DWI, Enhancement Features \\
\hline \multicolumn{3}{|c|}{ Suspected etiology $(n=29)$} \\
\hline Trauma & 1 & $\begin{array}{l}\text { History of trauma, imaging-associated sequelae of trauma, } \\
\text { subarachnoid hemorrhage, shear pattern }\end{array}$ \\
\hline Demyelinating & 1 & $\begin{array}{l}\text { Age, history of multiple sclerosis, periventricular/corpus } \\
\text { callosum predilection }\end{array}$ \\
\hline Hypoxic-ischemic & 5 & Hypotension, deep nuclei and cortical ribbon involvement \\
\hline Metastasis & 3 & History of malignancy and/or ring or enhancing lesion \\
\hline Seizure & 1 & Seizures, deep nuclei and/or cortical ribbon enhancement \\
\hline \multicolumn{3}{|l|}{ HIV-related $(n=2)$} \\
\hline Toxoplasmosis & 1 & $\begin{array}{l}\text { HIV, periventricular, ring, enhancing, target sign, rarely } \\
\text { shows restricted diffusion }\end{array}$ \\
\hline Fungal abscess & 1 & HIV, ring, enhancing, numerous restricted-diffusion lesions \\
\hline \multicolumn{3}{|l|}{ Cerebral emboli $(n=10)$} \\
\hline Endocarditis & 4 & Fever, leukocytosis, murmur, ring, enhancing \\
\hline Air & 1 & Followed esophagogastroduodenoscopy \\
\hline Fat & 1 & Followed long bone fracture \\
\hline Atrial fibrillation & 2 & No source other than atrial fibrillation found \\
\hline Aortic atheroma & 1 & Significant arch atheroma noted \\
\hline Aortic dissection & 1 & Patient had concomitant aortic dissection \\
\hline \multicolumn{3}{|l|}{ Postoperative $(n=6)$} \\
\hline Aneurysm coiling & 1 & Symptoms developed postoperatively \\
\hline Cardiac surgery & 4 & Symptoms developed postoperatively \\
\hline Aortic aneurysm repair & 1 & Symptoms developed postoperatively \\
\hline \multicolumn{3}{|l|}{ Not suspected $(n=10)$} \\
\hline Malignancy-related & 9 & $\begin{array}{l}\text { Lung }(n=4) \text {, colon }(n=2) \text {, renal }(n=1) \text {, pancreas }(n=1) \text {, } \\
\quad \text { bladder }(n=1)\end{array}$ \\
\hline Intravascular lymphoma & 1 & Proven by brain biopsy \\
\hline \multicolumn{3}{|l|}{ Indeterminate $(n=2)$} \\
\hline Incomplete history & 1 & Lost to follow-up \\
\hline Multiple etiologies & 1 & Possible fat emboli vs Trousseau syndrome \\
\hline
\end{tabular}

uated for radiographic appearance of DWI lesions. Lesions with MR characteristics of ischemic infarction (hyperintense on DWI and restricting on ADC) were deemed as infarction. The underlying etiology was determined from a chart review process. Lesion etiology was dichotomized into a "suspected" cohort (clinically suspected and documented or suggested by MR imaging report) and a "not suspected" cohort (clinically not suspected or documented and/or not suggested by radiology report) during hospitalization. The "not suspected" cohort represents the patients in whom etiology was not suspected by the treating physician or suggested by the radiologist because of the clinical history given and reported radiology reading.

\section{RESULTS}

Of 41 patients reviewed, a suspected etiology for 3-territory DWI lesions was identified in approximately 71\% $(n=$ 29 ), not suspected in approximately $24 \%(n=10)$, and indeterminate in approximately $5 \%(n=2)$. A total of 19 separate etiologies were identified, in-

nancy or vice versa, our patient selection was based on the presence of numerous, innumerable, multiple, or bilateral lesions on MR imaging. At our institution, we have experienced many cases of 3-cerebral territory infarctions associated with malignancy. However, the association of 3-territory DWI infarcts and malignancy has not been studied.

We speculate that selecting patients by using MR criteria allows for a more accurate assessment of diagnosing TS-related infarction compared with selection criteria using history of stroke and cancer because the potential for error exists when cancer history is overlooked or undiagnosed. In this study, we assessed the etiology of DWI-defined 3-territory infarcts, with attention to their diagnostic value in TS-related stroke.

\section{MATERIALS AND METHODS}

We sought to analyze the MR imaging characteristics of patients with DWI lesions involving 3 vascular territories (namely, the bilateral anterior and the posterior circulation) and correlate them with individual etiologies. A retrospective chart review at our institution was conducted after approval from the institutional review board for conducting research. All data on brain MR imaging conducted from July 1, 2014 to June 30, 2015 were included, encompassing 4075 studies with radiology reports. A search tool for the terms "restricted-diffusion" plus either "numerous," “innumerable," “multiple,” or "bilateral” yielded 709 studies. Of these studies, only reports with multiple DWI lesions that involved 3 vascular territories that included bilateral anterior and posterior circulation were analyzed. After exclusion, only 41 studies met criteria for analysis. Studies were reviewed and eval- cluding only 2 patients with indeterminate etiology. An embolic source, namely cardiac or large vessel, was the most common etiology in the "suspected" cohort. Of these 16 patients, only 1 had concomitant systemic malignancy. In the "not suspected" cohort of 10 patients, 9 had a concomitant systemic malignancy and 1 had intravascular lymphoma. In the absence of an identifiable embolic source, the presence of concomitant systemic malignancy was significantly associated with 3-territory DWI infarctions $(P<.001)$. The Table lists etiologies of all patients with 3 -territory DWI lesions with supporting clinical and radiographic features.

Moreover, patients with underlying malignancy represented approximately $29 \%(n=12)$ of all patients. In 9 patients, malignancy as cause of stroke etiology was diagnosed in a retrospective fashion after excluding all other potential causes and the concomitant presence of active malignancy. The mean age was $61 \pm 27$ years and $66 \%$ were men. The malignancies in our cohort included lung cancer $(n=4)$, colon cancer $(n=2)$, and 1 case each for renal, bladder, and pancreatic cancer. In only 2 patients, cause was indeterminate because of incomplete history or multiple possible etiologies. Although 1 patient with 3-territory infarctions during hospitalization was diagnosed with biopsy-proved intravascular lymphoma, it was not suspected by the treating physician or suggested by radiology report as the etiology.

In the "suspected" cohort, 3 patients had known metastatic cancer with 3-territory DWI involvement. However, lesions were ring-enhancing and suggestive of metastatic tumor spread to 


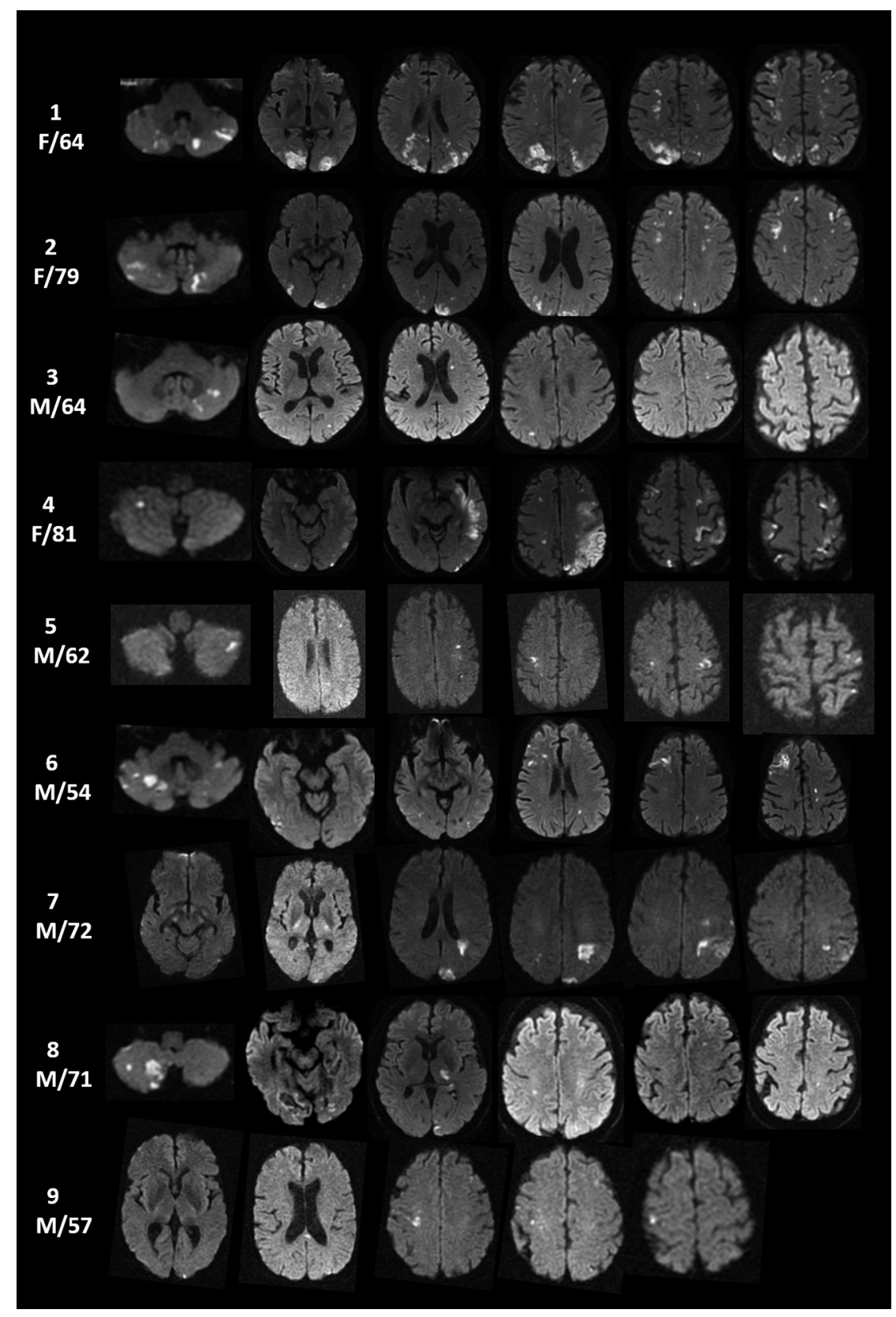

FIGURE. DWI infarcts involving bilateral anterior and posterior circulation (3 territory) with TS in 9 study group patients.

\section{DISCUSSION}

DWI infarction in multiple territories has been reported, ${ }^{4,10-12}$ including 3-territory lesions, ${ }^{4,10}$ yet a clear expression of the diagnostic significance of this MR imaging pattern with TS-related infarction has not been articulated. In our study, among the 12 patients without a suspected etiology, malignancy-related ischemic events were the source in 9 (75\%). Though not unique, the diffusion-weighted MR imaging features in our patients with malignancy-related infarction were highly suggestive.

In a prospective study evaluating embolic signals detected by transcranial Doppler in 74 patients with malignancyrelated infarction, embolic signals were more commonly seen in patients lacking conventional stroke risk factors $(P=$ .034) and were correlated strongly with D-dimer levels and the number of embolic signals detected $(P=<.001){ }^{13}$ The excess of embolic signal in patients with malignancy may explain the 3-territory DWI pattern observed or support this hypothesis, though this cannot exclude the possibility of intrinsic medium- and small-vessel thrombosis.

DWI lesions in our patient cohort also manifested diffuse changes that involve deep gray nuclei and/or cortical ribbon as seen with hypoxia-ischemia and seizure, whereas ring lesions, with or without enhancement, were associated with infection, metastasis, and endocarditis. Only endocarditis was associated with a peripheral predilection. TS-related infarction in the context of 3-territory DWI infarcts without an identifiable cause was not considered by the clinician or radiologist in our patients. Furthermore, in the presence of both malignancy and atrial fibrillation,

the brain. Other causes of 3-territory DWI lesions not defined as ischemic infarctions included infection, demyelination, seizure, and trauma. In the suspected cohort, an established embolic source was found in 10 patients and occurred postoperatively in 6 patients. Though the 3-territory pattern is the most compelling MR imaging feature of malignancy-related ischemic infarctions, characteristic radiographic DWI findings for malignancy-related infarction are often noted. These include nonenhancing, nonring clusters or single areas of restricted diffusion of $0.5-2 \mathrm{~cm}$ with a peripheral preference or large vascular territories, uncommonly in a watershed distribution, with absence of diffuse cortical ribbon or deep gray involvement (Figure). if echocardiogram shows no valvular pathology or intracardiac clot, TS should be a leading consideration. A tranesophageal echocardiogram is necessary to exclude a cardioembolic source.

In our study, we sought to evaluate the role of MR imaging in aiding with the diagnostic approach to multiple territory infarctions. Therefore, our approach was notably based on MR imaging as the identifying feature. We acknowledge that because of the retrospective nature of the study, linking the etiology of the 3-territory infarctions to malignancy is speculative based on excluding other causes as documented by the work-up completed by the treating physician. Moreover, it is possible that patients with true malignancy-related infarctions or TS involving only 1 or 2 territories were not accounted for in this study. Longitudinal data, 
such as prolonged cardiac monitoring to evaluate the possibility of atrial fibrillation in patients where etiology was not diagnosed during hospitalization, were not available. However, a substantial work-up was completed during hospitalization as 19 separate etiologies were identified.

Elevated D-dimer level, a direct measure of activated coagulation, is associated with malignancy and has been used as a measure of hypercoagulability in studies investigating stroke and malignancy. ${ }^{6,7}$ However, this biomarker is not specific to the hypercoagulable status of malignancy and can be elevated in several other conditions, such as infection, venous thromboembolism, and stroke. ${ }^{14} \mathrm{D}$-dimer levels were not reported in our study because of the few patients who had these levels tested during hospitalization. However, the aim of this study was to highlight the diagnostic value of the 3-territory DWI pattern as an additional diagnostic tool in the stroke work-up. In addition, because of the few patients in this study and the lack of patients with active malignancy and stroke of undetermined etiology involving 1 or 2 territories, the positive or negative predictive value of 3-territory DWI infarcts could not be calculated.

\section{CONCLUSIONS}

DWI infarcts involving 3 specific vascular territories, in the absence of an identifiable embolic source or other disease associated with such lesions, are highly suggestive of cancer-associated hypercoagulation stroke. Because TS-related stroke may be the heralding manifestation of an occult malignancy, work-up should include D-dimer and fibrinogen levels; tumor biomarkers; screening for deep vein thrombosis; CT of chest, abdomen, and pelvis; and PET scan when other tests are negative. Given the efficacy of heparin in preventing thrombotic events in cancerassociated hypercoagulation, timely diagnosis is paramount. As described here, the 3-territory DWI infarct pattern can provide an important diagnostic clue to an otherwise underrecognized cause of stroke. Future prospective studies evaluating patients with malignancy-related infarction with TS are needed to quantify the proportion of patients with 3-territory infarctions versus other MR patterns.

\section{REFERENCES}

1. Graus F, Rogers LR, Posner JB. Cerebrovascular complications in patients with cancer. Medicine 1985;64:16-35 CrossRef Medline

2. Taccone FS, Jeangette SM, Blecic SA. First-ever stroke as initial presentation of systemic cancer. J Stroke Cerebrovasc Dis 2008;17: 169-74 CrossRef Medline

3. Murinello A, Guedes P, Rocha G, et al. Trousseau's syndrome due to asymptomatic pancreatic adenocarcinoma. GE Port J Gastroenterol 2013;20:172-76 CrossRef

4. Kwon HM, Kang BS, Yoon BW. Stroke as the first manifestation of concealed cancer. J Neurol Sci 2007;258:80-83 CrossRef Medline

5. Varki A. Trousseau's syndrome: multiple definitions and multiple mechanisms. Blood 2007;110:1723-29 CrossRef Medline

6. Grisold W, Oberndorfer S, Struhal W. Stroke and cancer: a review. Acta Neurol Scand 2009;119:1-16 CrossRef Medline

7. Kim SG, Hong JM, Kim HY, et al. Ischemic stroke in cancer patients with and without conventional mechanisms: a multicenter study in Korea. Stroke 2010;41:798-801 CrossRef Medline

8. Schwarzbach CJ, Schaefer A, Ebert A, et al. Stroke and cancer: the importance of cancer-associated hypercoagulation as a possible stroke etiology. Stroke 2012;43:3029-34 CrossRef Medline

9. Chen Y, Zeng J, Xie X, et al. Clinical features of systemic cancer patients with acute cerebral infarction and its underlying pathogenesis. Int J Clin Exp Med 2015;8:4455-63 Medline

10. Kim SJ, Park JH, Lee MJ, et al. Clues to occult cancer in patients with ischemic stroke. PLoS One 2012;7:e44959 CrossRef Medline

11. Bang OY, Seok JM, Kim SG, et al. Ischemic stroke and cancer: stroke severely impacts cancer patients, while cancer increases the number of strokes. J Clin Neurol 2011;7:53-59 CrossRef Medline

12. Hong CT, Tsai LK, Jeng JS. Patterns of acute cerebral infarcts in patients with active malignancy using diffusion-weighted imaging. Cerebrovasc Dis 2009;28:411-16 CrossRef Medline

13. Seok JM, Kim SG, Kim JW, et al. Coagulopathy and embolic signal in cancer patients with ischemic stroke. Ann Neurol 2010;68:213-19 CrossRef Medline

14. Lippi G, Bonfanti L, Saccenti C, et al. Causes of elevated D-dimer in patients admitted to a large urban emergency department. Eur J Intern Med 2014;25:45-48 CrossRef Medline 